\title{
Are great disks defined by satellite galaxies in Milky-Way type halos rare in $\Lambda$ CDM?
}

\author{
X. Kang ${ }^{1}$, S. $\mathrm{Mao}^{2}$, L. Gao ${ }^{3}$, and Y. P. Jing ${ }^{1}$ \\ 1 Shanghai Astronomical Observatory; the Partner Group of MPA, Nandan Road 80, Shanghai 200030, PR China \\ e-mail: kangx@shao.ac.cn \\ 2 University of Manchester, Jodrell Bank Observatory, Macclesfield, Cheshire SK11 9DL, UK \\ 3 Max-Planck Institute for Astrophysics, Karl-Schwarzschild-strasse 1, Garching 85748, Germany
}

Received 17 January 2005 / Accepted 2 March 2005

\begin{abstract}
We study the spatial distribution of satellite galaxies by assuming that they follow the dark matter distribution. This assumption is supported by semi-analytical studies based on high-resolution numerical simulations. We find that for a Milky-Way type halo, if only a dozen satellite galaxies are observed, then they can lie on a "great" disk with an rms height of about $40 \mathrm{kpc}$. The normal to the plane is roughly isotropic on the sky. These results are consistent with the observed properties of the satellite galaxies in the Milky Way. If, however, the satellite galaxies follow the distribution of substructure selected by the present mass, then great disks similar to the one in the Milky Way will be rare and difficult to reproduce, in agreement with the conclusion reached by Kroupa et al. (2004).
\end{abstract}

Key words. Galaxy: evolution - Galaxy: halo - galaxies: dwarf - galaxies: structure

\section{Introduction}

The Cold Dark Matter (CDM) structure formation model has been successful in explaining many observations, in particular the large-scale structure of the universe (e.g. Peacock et al. 2001; Tegmark et al. 2004) and the cosmic microwave background (e.g., Spergel et al. 2003). However, on small scales, it appears to have problems in reproducing the rotation curves of dwarf galaxies and the satellite properties in Milky-Way type halos (e.g., see Silk 2004, for a review). But it is still a matter of debate whether these conflicts are real and whether they can be resolved within the CDM framework.

The CDM model generically predicts the existence of substructures (subhalos). In this model, larger structures form by merging of smaller structures, and dense cores in these small structures often survive the tidal force and manifest as substructures. These substructures are most clearly seen in highresolution numerical simulations (e.g., Klypin et al. 1999; Moore et al. 1999; Ghigna et al. 2000). If all these substructures host luminous galaxies, then the number of satellite galaxies in a Milky-Way type halo can reach several hundred, clearly exceeding the number of known satellite galaxies in the Milky Way (e.g., Kauffmann et al. 1993). But if only a small fraction $(\sim 10 \%)$ of the substructures form stars during their evolution, then the number of observed satellites can be reconciled with observations (Kravtsov et al. 2004). Whether the internal kinematics of satellite galaxies are consistent with the observations is still unclear (Stoehr et al. 2002; Kazantzidis et al. 2004).

Recently, Kroupa et al. (2004) highlighted the fact that the observed spatial distribution of satellite galaxies in the Milky Way is distributed in what they term a "great" disk; its plane is almost perpendicular to the plane of the Galactic disk. These properties are difficult to understand if the satellite galaxies follow the quasi-spherical distribution expected for the substructures. Recently, Gao et al. (2004a; see also Springel et al. 2001; Diemand et al. 2004; Nagi \& Kravtsov 2005) combined semianalytical techniques and high-resolution numerical simulations to examine the relation between substructures and (satellite) galaxies in clusters of galaxies. They showed that satellite galaxies follow roughly the same distribution as the underlying dark matter while the substructures follow a much shallower density distribution in the central part. In this paper, we show that if the satellite galaxies follow the same spatial distribution as dark matter, then many of their puzzling spatial properties highlighted by Kroupa et al. (2004) are easier to understand.

The outline of the paper is as follows. In Sect. 2 we first describe the simulation data we use, and then compare the spatial distribution of satellite galaxies with observations, concentrating on the statistics used by Kroupa et al. (2004). We discuss our results in Sect. 3. Throughout this paper, we adopt the "concordance" $\Lambda$ CDM cosmology (e.g., Ostriker \& Steinhardt 1995; Spergel et al. 2003, and references therein), with a matter density parameter $\Omega_{\mathrm{m}, 0}=0.3$, a cosmologically constant 
$\Omega_{\Lambda, 0}=0.7$, a baryon density parameter $\Omega_{\mathrm{b}}=0.024 h^{-2}$, and we take the power-spectrum normalization $\sigma_{8}=0.9$. We write the Hubble constant as $H_{0}=100 h \mathrm{~km} \mathrm{~s}^{-1} \mathrm{Mpc}^{-1}$ and adopt $h=0.7$.

\section{Method and results}

The data we use in this paper is the high resolution halo simulation of Jing \& Suto (2000). Four halos on galactic-mass scales (around $4 \times 10^{12} h^{-1} M_{\odot}$ ) were selected from a cosmological simulation with a box size of $100 h^{-1} \mathrm{Mpc}$. They were then re-simulated using the nested-grid PPPM code which was designed to simulate high-resolution halos. The force resolution is typically $0.4 \%$ of the virial radius, and each particle has a mass of about $6 \times 10^{6} h^{-1} M_{\odot}$ for the four galactic halos. We use the SUBFIND routine of Springel et al. (2001) to identify the disjointed self-bound subhalos within these halos and all subhalos with more than 10 particles are included in our analysis. Furthermore, in order to check the distribution of satellite galaxies in galactic halos, we use the galaxy catalog in a cosmological simulation with a box of $25 h^{-1} \mathrm{Mpc}$ constructed by Kang et al. (2004) using their semi-analytical model of galaxy formation. The sub-galactic satellites in their simulation were resolved with more than 10 particles, and each particle has mass of $7.7 \times 10^{7} h^{-1} M_{\odot}$.

It is known that in galaxy clusters the observed galaxy distribution follows the underlying dark matter (Gao et al. 2004a), but it is less clear whether the observed satellite galaxies in galactic mass halos follow the same distribution as the dark matter. In Fig. 1 we show the distribution of the observed satellites of the Milky Way and the comparisons with our simulation. The dotted line with triangles are the model satellite galaxies (with $M_{V}<-12$ ) in galactic halos in our simulation. The thick solid line is the distribution of dark matter in the high-resolution galactic halos. We found that the modeled satellite galaxies follow the underling dark matter distribution very well. Also it can be seen that the observed satellites roughly follow the dark matter particles. The Kolmogorov-Smirnov (KS) test shows that their distributions are consistent at a $60 \%$ confidence level. In contrast, the substructures with more than 10 particles clearly differ from the dark matter or the observed satellite distribution. In particular, they are not significantly centrally concentrated and have a higher density than observations in the outer part, as also found by others (De Lucia et al. 2004; Gao et al. 2004a; Diemand et al. 2004). Note that our subhalo population is incomplete as we can only identify those with more than ten particles $\left(>6 \times 10^{7} h^{-1} M_{\odot}\right)$. Increasing the mass resolution will result in more subhalos, but the relative number density profile will not change with the resolution as higher resolution primarily allows the measurement of the density profile near the centre (Diemand et al. 2004).

If all the subhalos form stars then the predicted spatial distribution of the satellites will be in conflict with the observed distribution. Furthermore, many models of galaxy formation will over-predict the number of dwarf satellite galaxies by an order of magnitude (Kauffmann et al. 1993; Bullock et al. 2000; Somerville 2002; Benson et al. 2002;

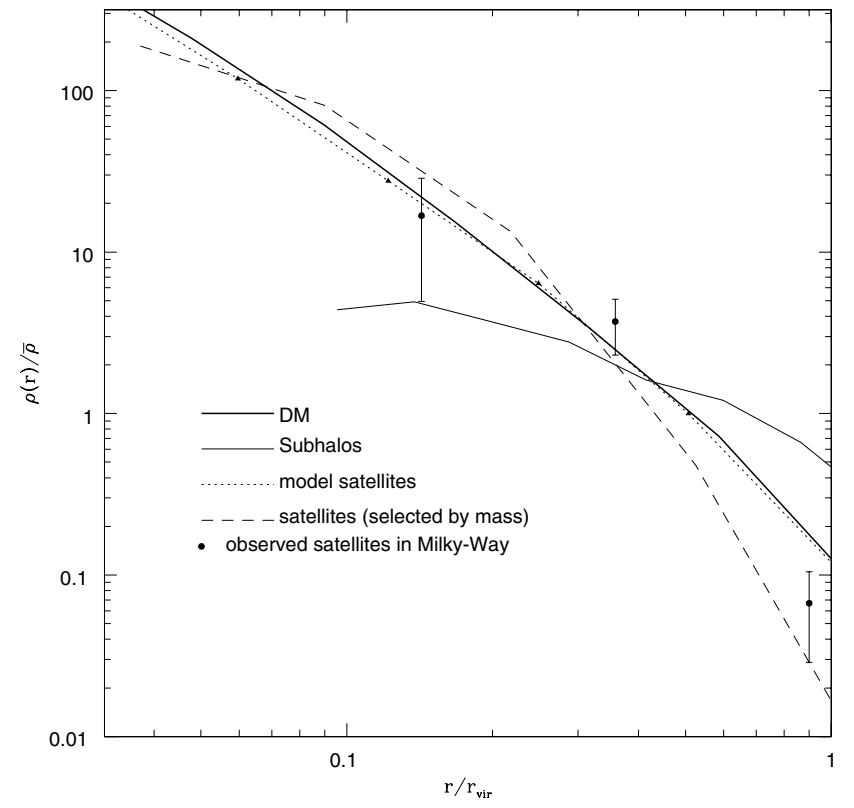

Fig. 1. The radial distribution of satellite galaxies. The solid circles are the distribution of the 11 inner satellites observed in the Milky Way (Mateo 1998; Kroupa et al. 2004); the Poisson error is indicated for each data point. The thick solid line is the dark matter profile and the thin solid line is for the substructures. The dotted line with triangles is for the satellite galaxies (brighter than $M_{V}=-12$ ) in galactic halos predicted by the semi-analytical model of Kang et al. (2004), and the dashed line is for the sample selected by mass at accretion (see text). Here the radius is normalized to the virial radius and the virial radius of the Milky Way is taken to be $250 \mathrm{kpc}$.

Kang et al. 2004). However, if there is a bias between the satellite galaxies and the subhalo population, then this overabundance problem can be alleviated (Taylor et al. 2003; Kravtsov et al. 2004). For example, using high-resolution $N$ body simulations, Kravtsov et al. (2004) showed that if only a fraction $(\sim 10 \%)$ of the subhalos with mass $\leq 10^{8}-10^{9} M_{\odot}$ host luminous galaxies, then the observed number of galactic satellites and their spatial distribution can be reproduced. They found that these luminous satellites are descendents of accreted massive halos $\left(\geq 10^{9} M_{\odot}\right)$ at $z \geq 2$. These massive systems with temperatures higher than $10^{4} \mathrm{~K}$ can undergo efficient gas cooling and star formation during their evolution. Motivated by this study, we check whether satellites (in a Milky Way type halo) selected in this way have a similar distribution as the observed satellites. There are about 100 massive halos $\left(\geq 10^{9} h^{-1} M_{\odot}\right)$ selected at accretion with redshift $z \geq 2$. The resolution of our re-simulated halos is not high enough for us to fully resolve the dwarf galaxies with mass around $10^{7} M_{\odot}$. We use the dynamical friction timescale given by Navarro et al. (1995, see their Eq. (2)) to determine which of the 100 most massive halos can survive in the final galactic halo, and we find that 30 of them survive. In order to obtain their final positions, we identify the most-bound particles of these halos at accretion and the final positions of the surviving halos at $z=0$ are tagged by these most-bound particles. This is reasonable as high-resolution $N$-body simulations have shown that the inner 
part of the halo will remain intact during the evolution (e.g. Springel et al. 2001). The radial distribution of these surviving halos is plotted in Fig. 1 with the dashed line. Clearly the satellite galaxies selected by mass at accretion have a similar distribution as the observed satellites in the Milky Way. The KS test shows that it also agrees with the radial distribution of the dark matter particles at a $20 \%$ confidence level. We also check if the satellites selected in this way have a similar shape with the dark matter particles. The number of satellites (30) is not large enough to define their shape accurately. Nevertheless, we fit their distribution with a triaxial distribution and characterize their shape as the ratio between the minor and major axes. We then use a Monte Carlo simulation to produce a distribution of such ratios by selecting the satellites from the underlying dark matter particles. We found that the ratio of the satellites selected by mass at accretion lies at the $75 \%$ percentile of the distribution. This agreement provides the justification to select the satellites galaxies from the dark matter particles in the re-simulated galactic halos. We also consider the case where the satellite galaxies follow the distribution of the subhalos $\left(\geq 6 \times 10^{7} h^{-1} M_{\odot}\right)$, but we will show that their distribution is not consistent with the observations.

To examine the spatial distribution of satellites in our simulated halos, we use Monte Carlo simulations to select the satellites assuming they follow either the dark matter distribution or the substructure distribution. For a full comparison with the results of Kroupa et al. (2004), we selected 11 random satellites in a sphere with a radius of about $250 \mathrm{kpc}$ from the centre. In order to see how the results change with the number of satellites, we also show the results obtained by increasing the number of satellites by a factor of 2, i.e., 22 satellites within the same sphere.

Following Kroupa et al. (2004), we fit the selected satellites with a plane by minimizing the rms of the height. The thickness of the plane is the minimum of the rms of the height $\Delta$. The ratio of $\Delta$ to the maximum distance $\left(R_{\text {cut }}\right)$ to the satellites is used to characterize the thickness of the plane. In Fig. 2 we show the distribution of the characteristic thickness $\Delta / R_{\text {cut }}$ of the fitted plane from a large number of Monte Carlo realizations. The four panels are for the four different halos. In these plots the histograms without symbols are the distributions of the satellites selected from the dark matter particles and those with symbols are for the substructures. We find that for dark matter samples, the thickness of the planes are smaller than that in the samples of substructures. The average thickness of the planes peaks at $\Delta / R_{\text {cut }} \sim 0.17$. It can also be seen that increasing the number of satellites also increases the thickness of the planes. Kroupa et al. (2004) showed that for the inner 11 satellites the characteristic thickness of the plane is $\Delta / R_{\text {cut }} \approx 0.1$, but this $\Delta / R_{\text {cut }}$ value changes when one adopts a different number of satellites. For example, for the 9 innermost satellites, the characteristic thickness of the plane is 0.17 . The thick horizontal bar in the top left panel of Fig. 2 indicates the range of $\Delta / R_{\text {cut }}$ if one adopts 5-16 satellite galaxies in the Milky Way. Clearly it is difficult to produce a value as small as the observed one if the satellite galaxies follow the substructure distribution. In contrast, if the satellite galaxies follow the dark matter distribution, it is not difficult to obtain the observed low

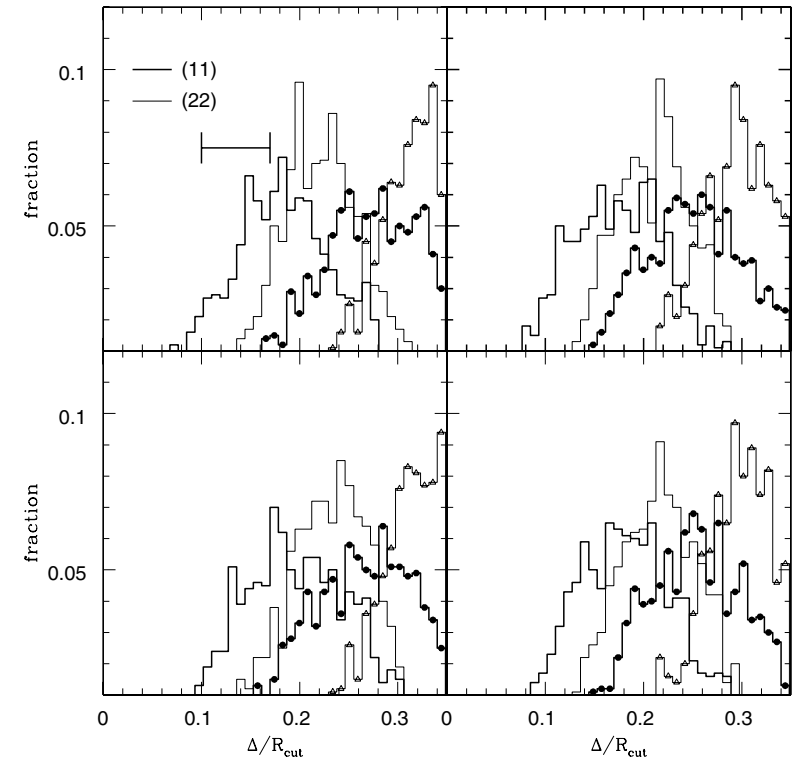

Fig. 2. The distribution of the rms of the scale height from a plane defined by satellite galaxies obtained through many Monte Carlo realizations. The four panels are for four different galactic-sized halos. The histograms without symbols show the result expected if the satellite galaxies follow the dark matter distribution while the histograms with symbols show those if satellites follow the subhalo distribution. The thick solid histograms are for 11 satellite galaxies, while the thin solid histograms are for 22 satellite galaxies. The horizontal bar in the top left panel shows the range of $\Delta / R_{\text {cut }}$ for the observed satellites when different numbers of satellite galaxies are used (see Table 1 in Kroupa et al. 2004).

value of $\Delta / R_{\text {cut }}$. It is easy to show that for a given spherical density profile $\rho \propto r^{-n}$, the thickness of the plane as defined here is given by

$\frac{\Delta}{R_{\text {cut }}}=\sqrt{\frac{3-n}{3(5-n)}}, n<3$.

The above equation is valid when all the particles in the sphere are selected. Notice also that when the power-law index $n \rightarrow 3$, the thickness goes to zero. This is because the mass and hence the particle number diverge at the origin when $n \rightarrow 3$. The plane determined by these (infinite) number of particles around the origin formally will have zero thickness.

From Fig. 1 we see that the substructures have a shallower slope (i.e., a smaller value of $n$ ) at the centre, so most of them are in the outer part of the halo, which makes the fitted plane thicker. The observed satellites, in contrast, follow a steeper slope than the substructures, so satellites are more likely to lie in the inner part of the halo and hence make the plane more prominent. The thickness also depends on the number of particles selected. Clearly a plane with only 3 particles will have zero thickness while a plane with an infinite number of particles will have a thickness given by Eq. (1). This is why a larger characteristic thickness is found when we increase the satellite number from 11 to 22 in Fig. 2.

Kroupa et al. (2004) point out that the fitted plane of the Milky Way satellites is almost perpendicular to the Galactic disk; the normals to these two planes have an angle of 


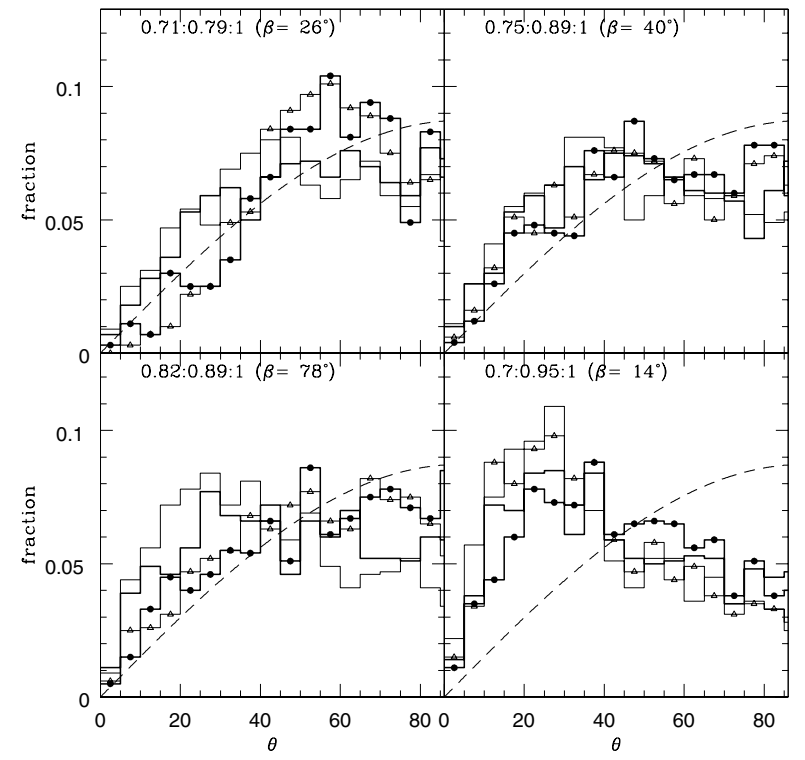

Fig. 3. The distribution of the rms of the angle (in degrees) between the plane defined by satellite galaxies and the plane defined by the major and medium axes in the triaxial halo model (see text). The dashed line is the prediction if the angle between the satellite plane and the minor axis of the halo is randomly distributed on the sky. The other line symbols are the same as in Fig. 2. In each panel we label the ratio of the minor, medium and maximum axes. In parentheses we indicate the angle between the angular momentum vector and the minor axis of the triaxial model.

approximately $75^{\circ}$. Unfortunately it is not clear how we should define the disk plane in our simulated galaxies. There are at least two ways of defining the disk plane. One is simply to identify the total angular momentum vector as the normal to the disk plane. The second way is to fit the density profile of the halos with a triaxial distribution, obtain the three principal vectors ( $\boldsymbol{a}, \boldsymbol{b}$ and $\boldsymbol{c}, a \leq b \leq c)$, and identify the disk plane as that defined by the major and medium axes. These two definitions would be the same if the minor axis is parallel to the angular momentum vector. While there is a statistical correlation between these two, the scatter is quite large (e.g., Faltenbacher et al. 2002). In Fig. 3 we show the angle $(\beta)$ between the total angular momentum vector and the minor axis. Note that we use all the particles enclosed within the virial radius to obtain the total angular momentum vector and the triaxial density profile. As one can see, the alignment for three halos is within $40^{\circ}$, but for the halo in the lower left panel, $\beta=78^{\circ}$, i.e., the minor axis is almost perpendicular to the total angular momentum vector. The identification of the disk plane is further complicated by the fact that it is not clear how the angular momentum of the (baryonic) disk is related to the total angular momentum of the dark matter in $\mathrm{N}$-body/hydro-dynamical simulations. Chen et al. (2003) showed that there is a large scatter between these two.

In Fig. 3 we show the distribution of the angle $(\theta)$ between the normal vector to the fitted plane and the minor axis $\boldsymbol{a}$, assuming that the disk plane is the same as the plane defined by the major and medium axes. In each panel the ratio of $a: b: c$ is also labeled. For two randomly oriented normal vectors, the

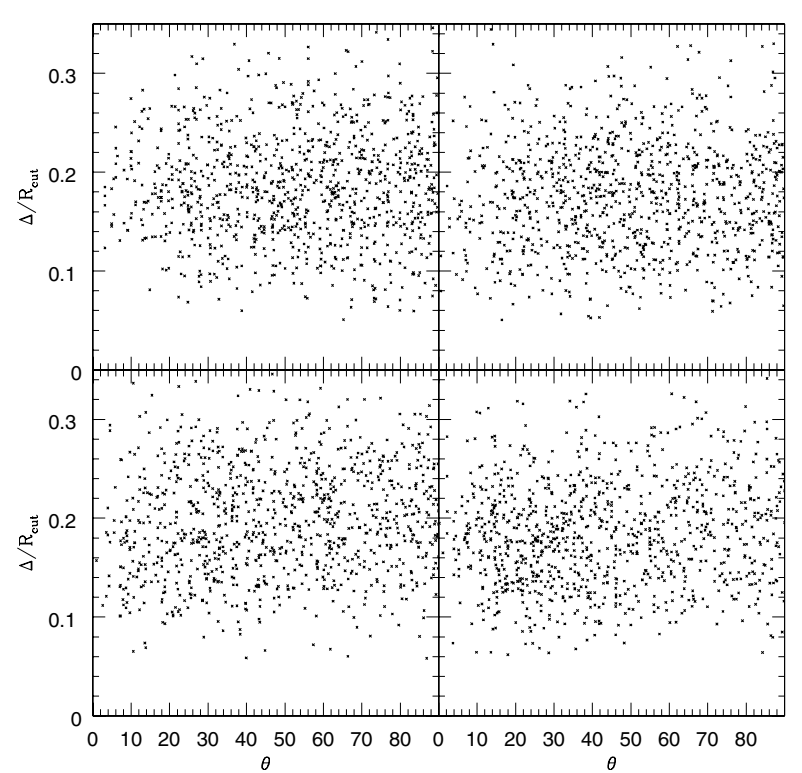

Fig. 4. A scatter plot of $\theta$ vs. $\Delta / R_{\text {cut }}$ for the satellites selected from the dark matter particles. $\theta$ is the same as that in Fig. 3.

probability distribution for the angle between them is $\propto \sin \theta$, which is shown as the dashed line in Fig. 3. Compared with a random distribution, the satellites are preferentially located in a plane defined by the major and medium principal axes. If we select only 11 satellite galaxies, then the probabilities for the four halos to have an angle $\theta$ larger than $60^{\circ}\left(80^{\circ}\right)$ are between $28-41 \%(9 \%-14 \%)$, respectively. Thus, the probability for the fitted satellite plane to have a large angle with the galaxy disk is not negligible. On the other hand, if we identify the galaxy disk as that given by the angular momentum vector, these corresponding probabilities become even larger, $28 \%-70 \%(9 \%-26 \%)$ for $\theta>60^{\circ}\left(\theta>80^{\circ}\right)$.

The above results show that there is some probability for the selected satellite galaxies to lie in a plane and some probability for the plane to be perpendicular to the disk. However it is important to examine the joint distribution of $\Delta / R_{\text {cut }}$ and the angle $\theta$. Figure 4 shows the scatter plot between $\Delta / R_{\text {cut }}$ and the angle $\theta$ for the satellites from the dark matter distribution (the scatter plot for substructures is similar, but of course with larger values of $\Delta / R_{\text {cut }}$ ). As we can see, there is no correlation between them; for a given thickness of the plane, it has roughly the same probability of having large and small $\theta$ values. The probability of having $\theta>60^{\circ}\left(80^{\circ}\right)$ and $\Delta / R_{\text {cut }}<0.17$ ranges from $10 \%$ to $17 \%$ (from $3 \%$ to $6 \%$ ) for the four different haloes. This means that it is not rare for the observed plane to have large inclinations to the disk.

Kroupa et al. (2004) have analyzed the cumulative distribution of $\cos \omega$ for the Milky Way satellites, where $\omega$ is the angle between the normal to the great disk and the vector connecting the point on the great disk that is closest to the Galactic centre to the position of the satellite. By design, the distribution of $\cos \omega$ is closely related to the thickness of the great disk. Kroupa et al. (2004) compared this distribution to that of $10^{5}$ points derived from a spherical power-law density distribution $r^{-n}$. Using the KS test, they found the likelihood, $P_{\mathrm{ks}}$, that the observed Milky Way distribution is derived from the 
$r^{-n}$ model is only 0.005 for $0<n<2.3$. Taken at face value, this seriously challenges the assumption that the Milky Way satellites are consistent with an isotropic parent distribution.

We have repeated their analysis for the Milky Way, and found that $P_{\mathrm{ks}}$ is $\sim 0.01$ if the distribution of $|\cos \omega|$ is used, consistent with Kroupa et al. (2004), who used the same quantity although they imply that a different quantity, $\cos \omega$, was used (Kroupa 2005, private communication). There is no obvious reason why one should use $|\cos \omega|$ instead of $\cos \omega$. The distribution of $\cos \omega$ should equally well describe the thickness of the great disk. When we use $\cos \omega$, we found that $P_{\mathrm{ks}}$ increases dramatically to $\sim 20 \%$ for the Milky Way satellites, which implies that the great disk is compatible with the hypothesis that the satellites are drawn from an isotropic parent population.

We randomly select 11 dark matter particles from the first galactic halo, and repeat the above KS test for the simulation satellites. We have made many realizations, and found that there is a $32 \%(8.8 \%)$ probability that the $P_{\mathrm{ks}}$ of the simulated satellites is smaller than that of the observed $\cos \omega(|\cos \omega|)$ distribution for the Milky Way. About 40\% (19\%) of the simulated populations have $P_{\mathrm{ks}}<0.1$ for the $\cos \omega(|\cos \omega|)$ distributions. These results have two important implications. First, the KS probability using $|\cos \omega|$ (or $\cos \omega$ ) should not be directly interpreted as the probability of whether the satellites are consistent with being drawn from a spherical $r^{-n}$ density distribution, otherwise the probability may be under-estimated. This is because the great disk is determined using the positions of all the satellite galaxies, and as a result the $\cos \omega$ values based on the great disk are no longer independent of each other. This makes the KS probability misleading as it assumes that the $\cos \omega$ values are independent data points (e.g., Lupton 1993). The following is an extreme example that illustrates this point clearly. If only 3 simulated satellites are selected from a spherical $r^{-n}$ distribution, then $\cos \omega$ is zero for all the three satellites. One finds that the KS probability defined above is very small $(\sim 0.13 \%)$. This low probability is clearly misleading as this population is drawn from an isotropic power-law density distribution. Also, our results clearly show that there is a $32 \%$ (or $8.8 \%$ ) probability of reproducing the observed $\cos \omega$ (or $|\cos \omega|$ ) distribution in the $\Lambda \mathrm{CDM}$ halos, thus it is not so rare to expect great disks similar to the one in the Milky Way in the $\Lambda$ CDM model.

\section{Discussion}

The presence of dark matter substructures is a generic prediction of the $\Lambda \mathrm{CDM}$ model. However, as pointed out by many authors (e.g., Springel et al. 2001; Gao et al. 2004a,b; Nagi \& Kravtsov 2005), the correspondence between dark matter substructures and luminous satellite galaxies is not simple. This is because the stellar mass of a galaxy may be primarily determined when it is first formed. In contrast, the dark matter substructure may evolve quite differently due to dynamical friction and other processes at later times. In the study of Gao et al. (2004a), they find $\sim 50 \%$ of satellite galaxies have no corresponding dark matter subhalos.

If the satellite galaxy distribution follows the dark matter distribution, our Monte Carlo simulations demonstrate that the spatial distribution of satellite galaxies can be better accommodated within the $\Lambda \mathrm{CDM}$ cosmogony. In particular, the fact that the Milky Way satellite galaxies are distributed in a great disk with its plane almost perpendicular to the stellar disk is not as rare as one naively may expect. In particular, for the halo shown in the bottom left panel of Fig. 3, as the angular momentum vector of the halo is almost perpendicular to minor axis, it has the largest probability, $>26 \%$ (or 14\%) for the fitted plane to have an angle larger than $80^{\circ}$ to the disk if we identify the disk plane as that given by the total angular momentum vector (or the minor axis in the triaxial model).

In contrast, if the satellite galaxies follow the distribution of the substructures selected by the present-day mass then we find that the satellite galaxy properties will be difficult to match because the substructures have a flatter slope with $n \sim 0$. But if only $\sim 10 \%$ percent of the substructures host luminous galaxies, the observed distribution of the satellite galaxies can be recovered, as also shown by Kravtsov et al. (2004). However it remains somewhat puzzling as to why the observed "great" disk is almost perpendicular to the disk. As we have shown at the end of Sect. 2, the probability of having a thin great disk perpendicular to the Galactic plane is of a few percent to $\sim 17 \%$. In our simulations, we find that the the fitted plane of satellite galaxies have a larger probability of lying in the disk defined by the major and medium axes in the triaxial model. If the baryonic disk is in the same plane, then the great disk in the Milky Way is most easily explained if the Galactic plane has a large inclination to the plane defined by the the major and medium axes of the dark matter distribution. Satellite streams such as Sagittaris can in principle probe the shape of the halo. However, the current evidence is still not yet conclusive (e.g. Helmi 2004a,b; Johnston et al. 2004).

Unfortunately, the comparison between our simulations and observations is not direct. Theoretically, with our collisionless, dark matter only simulations, it is not clear how to relate the dark matter properties (such as the angular momentum vector) to baryonic disk properties. Furthermore, the dark matter halo and satellite galaxy profiles will be affected (perhaps adiabatically, e.g., Mo et al. 1998) by the assembly of the baryonic disk. Furthermore, the presence of the disk may force subhalos into roughly coplanar orbits (Meza et al. 2004) and makes it more difficult to have the satellite great disk perpendicular to the stellar disk. Observationally, it is not clear if all the satellite galaxies in the Milky Way have been found. In particular, some satellite galaxies in low Galactic latitudes may have been missed (Willman et al. 2004). The addition of such satellites (if at large distances) will make the great disk in the Milky Way much thicker. It appears that the issue of satellite galaxies can only be fully resolved when more observations and highresolution hydrodynamical simulations become available.

Acknowledgements. We thank the referee, Dr. Juerg Diemand, for a helpful report and Dr. P. Kroupa for clarifications. S.M. and L.G. thank the Shanghai Astronomical Observatory for hospitality during several visits. S.M. acknowledges the financial support of the Chinese Academy of Sciences and the EU ANGLES research network. The research is supported by NKBRSF (G19990754), by NSFC (Nos. 10125314, 10373012), and by Shanghai Key Projects in 
Basic research (No. 04jc14079). X.K. is supported in part by NSFC (10203004).

\section{References}

Benson, A. J., Frenk, C. S., Lacey, C. G., Baugh, C. M., \& Cole, S. 2002, MNRAS, 333, 177

Bullock, J. S., Kravtsov, A. V., \& Weinberg, D. H. 2000, ApJ, 539, 517

Chen, D. N., Jing, Y. P., \& Yoshikaw, K. 2003, ApJ, 597, 35

Diemand, J., Moore, B., \& Stadel, J. 2004, MNRAS, 352, 535

Faltenbacher, A., Gottlöber, S., Kerscher, M., \& Müller, V. 2002, $A \& A, 395,1$

Gao, L., De Lucia, G., White, S. D. M., \& Jenkins, A. 2004a, MNRAS, 352, L1

Gao, L., White, S. D. M., Jenkins, A., Stoehr, F., \& Springel, V. 2004b, MNRAS, 355, 819

Ghigna, S., Moore, B., Governato, F., et al. 2000, ApJ, 544, 616

Helmi, A. 2004a, MNRAS, 351, 643

Helmi, A. 2004b, ApJ, 610, L97

Ibata, R., Irwin, M., Lewis, G. F., \& Stolte, A. 2001, ApJ, 547, L133

Jing, Y. P., \& Suto, Y. 2000, ApJ, 529, L69

Johnston, K. V., Law, D. R., \& Majewski, S. R. 2005, ApJ, 619, 800

Kang, X., Jing, Y. P., Mo, H. J., \& Börner, G. 2004, ApJ, submitted [arXiv:astro-ph/0408475]

Kauffmann, G., White, S. D. M., \& Guiderdoni, B. 1993, MNRAS, 264,201
Kazantzidis, S., Mayer, L., Mastropietro, C., Diemand, J., Stadel, J., \& Moore, B. 2004, ApJ, 608, 663

Klypin, A., Kravtsov, A. V., \& Valenzuela, O. 1999, ApJ, 522, 82

Kravtsov, A. V., Gnedin, O. Y., \& Klypin, A. A. 2004, ApJ, 609, 482

Kroupa, P., Theis, C., \& Boily, C. M. 2004, A\&A, in press

Lupton, R. H. 1993, Statistics in theory and practice (Princeton: Princeton University Press), 120

Mateo, M. 1998, ARA\&A, 36, 435

Meza, A., Navarro, J. F., Abadi, M. G., \& Steinmetz, M. 2004, preprint [arXiv: astro-ph/0408567]

Mo, H. J., Mao, S., \& White, S. D. M. 1998, MNRAS, 295, 319

Moore, B., Ghigna, S., Governato, F., et al. 1999, ApJ, 524, L19

Nagai, D., \& Kravtsov, A. V. 2005, ApJ, 618, 557

Navarro, J. F., Frenk, C. S., \& White, S. D. M. 1997, ApJ, 490, 493

Peacock, J. A., Cole, S., Norberg, P., et al. 2001, Nature, 410, 169

Ostriker, J. P., \& Steinhardt, P. J. 1995, Nature, 377, 600

Silk, J. 2004, preprint [arXiv: astro-ph/0412297]

Somerville, R. S. 2002, ApJ, 572, 23

Springel, V., White, S. D. M., Tormen, G., \& Kauffmann, G. 2001, MNRAS, 328, 726

Spergel, D. N. S., Verde, L., Peiris, H. V., et al. 2003, ApJS, 148, 175

Stoehr, F., White, S. D. M., Tormen, G., \& Springel, V. 2002, MNRAS, 335, L84

Tegmark, M., Blanton, M. R., Strauss, M. A., et al. 2004, ApJ, 606, 702

Willman, B., Governato, F., Dalcanton, J. J., Reed, D., \& Quinn, T. 2004, MNRAS, 353, 639 